\title{
Multidrug-resistant Gram-negative bacteria-resistant infections: epidemiology, clinical issues and therapeutic options
}

\author{
Matteo Bassetti, Davide Pecori, Maddalena Peghin \\ Infectious Diseases Clinic, Santa Maria Misericordia University Hospital, Udine, Italy
}

\begin{abstract}
In the last decade, we have witnessed a dramatic increase in the number of multidrug resistant Gram-negative (MDRGN) bacterial pathogens, both in Italy and worldwide, with Enterobacteriacae (mostly Klebsiella pneumoniae), Pseudomonas aeruginosa and Acinetobacter baumannii being the major threats in clinical practice. Inadequate empirical antimicrobial therapy of severe infections caused by MDR Enterobacteriacae has been associated with an increased morbidity and mortality. However, a careful selection of patients who may receive empirical treatment covering MDR Enterobacteriacae is important to avoid the overuse of broad-spectrum antibiotics. The aim of this review is to describe the mechanism of resistance, epidemiology, risk factors, clinical issues, and therapeutic options for MDRGN pathogens.
\end{abstract}

\section{Introduction}

In the last decade, we have witnessed a dramatic increase worldwide in the number of multidrug resistant Gram-negative (MDRGN) bacterial pathogens, with Enterobacteriacae (mostly Klebsiella pneumoniae), Pseudomonas aeruginosa and Acinetobacter baumannii being the major threats in clinical practice. Due to the resistance to the most common antibiotics prescribed as empiric regimens, MDRGN bugs have been associated with delays in an adequate treatment, leading to significant increases in morbidity and mortality. ${ }^{1}$ In addition, the spread of MDRGN pathogens resulted over last years in a vicious circle of an indiscriminate prescription of broad-spectrum antimicrobials and further resistance selection. ${ }^{2}$

Correspondence: Matteo Bassetti, Infectious Diseases Clinic, Santa Maria Misericordia University Hospital, Piazzale Santa Maria della Misericordia 15, 33100 Udine, Italy. Tel.: +39.0432.559353 - Fax: +39.0432 .559360 .

E-mail: mattba@tin.it

Key words: Enterobacteriaceae; extended-spectrum $\beta$-lactamase; Klebsiella pneumoniae carbapenemase; multidrug-resistant; treatment.

Received for publication: 6 October 2016.

Revision received: 30 October 2016.

Accepted for publication: 3 November 2016.

This work is licensed under a Creative Commons Attribution NonCommercial 4.0 License (CC BY-NC 4.0).

CCopyright M. Bassetti et al., 2016

Licensee PAGEPress, Italy

Italian Journal of Medicine 2016; 10:364-375

doi:10.4081/itjm.2016.802
The aim of this review is to describe the mechanism of resistance, epidemiology, risk factors, clinical issues, and therapeutic options of MDRGN pathogens.

\section{Mechanism of resistance of Gram-negative bacteria}

MGRGN bacteria are defined as pathogens carrying resistance to one or more antimicrobials from at least three different classes. The most common mechanism of resistance is represented by intrinsic and acquired production of $\beta$-lactamases, which can be chromosomal or plasmid mediated. $\beta$-lactamases are hydrolytic enzymes able to disrupt the $\beta$-lactam ring, thus inactivating different classes of $\beta$-lactams. ${ }^{3,4}$ The most common enzymes in clinical practice are the extended-spectrum- $\beta$-lactamases (ESBLs), which are mostly expressed by Enterobacteriaceae. A novel type of class $C \beta$-lactamases also showing activity against cefepime and denominated extended spectrum AmpC $\beta$-lactamases has been described. ${ }^{4}$

The consequent abuse of carbapenems, representing the first choice for ESBL infections, led to a progressive increase in carbapenem resistance, mainly due to the production of carbapenem-hydrolyzing $\beta$-lactamases, or carbapenemases, that usually confer clinical resistance to most $\beta$-lactams. ${ }^{5,6} \mathrm{~K}$. pneumoniae carbapenemases (KPCs) are the most relevant enzymes among Enterobacteriaceae, and confer resistance to all the $\beta$ lactams, including $\beta$-lactam $/ \beta$-lactamase inhibitors combinations. Class B enzymes, named metallo- $\beta$-lactamases are expressed by both enterics and $P$. aeruginosa and confer resistance to all $\beta$-lactams with the exception of aztreonam. Oxacillinases belong to the class D $\beta$-lactamases, and are mostly expressed in $P$. 
aeruginosa and $A$. baumannii. ${ }^{7}$ Table 1 summarizes the classification of $\beta$-lactamases. ${ }^{3}$

Together with the production of $\beta$-lactamases, additional mechanisms of carbapenem-resistance are the down-regulation of porins and efflux pumps, the last most common in $P$. aeruginosa and the expression of additional genes harbouring resistance to other antimicrobial classes, such as fluoroquinolones and aminoglycosides, thus narrowing the spectrum of therapeutic options. ${ }^{8}$

\section{Epidemiology of emerging multidrug resistant Gram-negative bacteria in Italy}

The burden of MDRGN bacteria has alarmingly increased worldwide over last decade, even with a wide variability between different countries. ${ }^{9}$ In Europe, the distribution of antimicrobial resistance is widely jeopardized, but the Annual report of the European Antimicrobial Resistance Surveillance Network (EARS-Net) showed a generalized increase in antimicrobial resistance particularly in Southern regions. ${ }^{10}$

Focusing on the Italian epidemiological setting, more than $50 \%$ of $K$. pneumoniae isolates showed resistance to third-generation cephalosporins, and the large majority of isolates (85-100\%) expressed ESBL enzymes. In Escherichia coli, a combined resistance to third-generation cephalosporins, fluroquinolones and aminoglycosides was reported in $10-25 \%$ of cases.
Carbapenem-resistance represents nowadays the most alarming problem for our country. Among Enterobacteriaceae, the major threat comes from $K$. pneumoniae, since in 2014 in Italy approximately 25 $50 \%$ of isolates were resistant to carbapenems, compared to less than $5 \%$ of isolates in 2009 . Conversely, in $E$. coli carbapenem-resistant is currently reported in less than $5 \%$ of cases, but represents an emerging problem in clinical practice. ${ }^{11}$

P. aeruginosa also represents a worrisome problem. A significant increase in resistance to piperacillin/ tazobactam has been observed (around 17\% in 2014 in Europe). Moreover, in Italy, approximately $25-50 \%$ of $P$. aeruginosa isolates showed resistance to carbapenems and up to $10-50 \%$ of strains were classified as MDR. Similar data have been reported in A. baumannii, which showed combined resistance to fluoroquinolones, aminoglycosides and carbapenems in up to $50 \%$ of cases, with an alarming increase in colistin-resistance, reported in approximately $4 \%$ of isolates, mostly in Italy. Table 2 summarizes epidemiology of MDRGN bacteria worldwide and in Italy.

\section{Risk factors, clinical issues and empirical treatment for emerging multidrug resistant Gram-negative infections}

Emerging MDRGN pathogens are related both to individual risk factors and to local epidemiological

Table 1. Summary of relevant, emerging broad-spectrum, plasmid-mediated $\beta$-lactamases in multidrug-resistant Gramnegative bacteria.

\begin{tabular}{|c|c|c|c|}
\hline Molecular class & Enzymes & Spectrum & Epidemiology \\
\hline \multirow[t]{2}{*}{ A } & $\begin{array}{l}\text { Extended-spectrum } \beta \text {-lactamases } \\
\text { (TEM, SHV, CTX-M, others) }\end{array}$ & $\begin{array}{l}\text { Penicillins, cephalosporins } \\
\text { (except cefamycins), aztreonam } \\
\text { Inhibited by } \beta \text {-lactamase inhibitors }\end{array}$ & $\begin{array}{l}\text { Worldwide spread: USA, Greece, Italy, } \\
\text { Israel, China } \\
\text { Community and nosocomial infections }\end{array}$ \\
\hline & $\mathrm{KPC}$ & $\begin{array}{l}\text { Penicillins, cephalosporins, } \\
\text { aztreonam, carbapenems. Inhibited } \\
\text { by } \beta \text {-lactamase inhibitors }\end{array}$ & $\begin{array}{l}\text { Mainly nosocomial outbreaks } \\
\text { Frequent in some areas of USA, Greece, } \\
\text { Israel, Italy, etc. }\end{array}$ \\
\hline B & $\begin{array}{l}\text { Metallo } \beta \text {-lactamases (VIM, IMP, } \\
\text { NDM, others) }\end{array}$ & $\begin{array}{l}\text { Penicillins, cephalosporins and } \\
\text { carbapenems } \\
\text { Monobactams are susceptible } \\
\text { Not inhibited by } \beta \text {-lactamase } \\
\text { inhibitors }\end{array}$ & $\begin{array}{l}\text { Worldwide spread: Greece, Italy, Indian } \\
\text { subcontinent, Balkans, Middle East } \\
\text { Nosocomial outbreaks and endemic } \\
\text { situations }\end{array}$ \\
\hline $\mathrm{C}$ & $\begin{array}{l}\text { AmpC type (CMY-2, DHA-1, } \\
\text { FOX-1, others) }\end{array}$ & $\begin{array}{l}\text { Penicillins, cephalosporins } \\
\text { (except cefepime), and } \\
\text { monobactams } \\
\text { Not inhibited by } \beta \text {-lactamase } \\
\text { inhibitors }\end{array}$ & $\begin{array}{l}\text { Worldwide spread } \\
\text { Community and nosocomial infections }\end{array}$ \\
\hline $\mathrm{D}$ & OXA (OXA-48, OXA-23, others) & $\begin{array}{l}\text { Penicillin, aztreonam and } \\
\text { carbapenems } \\
\text { Not inhibited by } \beta \text {-lactamase } \\
\text { inhibitors }\end{array}$ & $\begin{array}{l}\text { Nosocomial outbreak } \\
\text { Spread in Middle East, Mediterranean } \\
\text { countries (Spain) and Africa }\end{array}$ \\
\hline
\end{tabular}

TEM, temoniera (a patient from whom the strain was first isolated in Greece); SHV, sulphydryl variable; CTX-M, cefotaxime hydrolyzing capabilities; KPC, Klebsiella pneumoniae carbapenemase; VIM, Verona integron-encoded metallo- $\beta$-lactamase; IMP, imipenem-type metallo- $\beta$-lactamase; NDM, New Delhi metallo- $\beta$-lactamase; CMY-2, cephamycins; DHA1, Dhahran Hospital; FOX-1, cefoxitin; OXA, oxacillinase. Modified from Delgado-Valverde et al., 2013. ${ }^{4}$ 
background, including community, long-term care facilities or hospital setting. While ESBL Enterobacteriaceae are found in the community and hospital environment, carbapenemase-producing Enterobacteriacae (CRE), MDR P. aeruginosa and MDR A. baumannii are still mainly found in hospitalized patients, although OXA-48 CRE might be isolated in the community. ${ }^{12}$ In clinical practice, Intensive Care Unit (ICU) setting has been traditionally considered the tip of the iceberg of hospital MDRGN infections. ${ }^{13-15}$ However, beside ICU setting, in recent years the spread of MDRGN pathogens has been increasingly described also in other settings, such as oncologic and hematologic wards ${ }^{16,17}$ and long-term-care facilities. ${ }^{18,19} \mathrm{~A}$ bedridden status, presence of indwelling devices, recent hospitalization or contact with health care facilities and recent antibiotic therapy may represent the most important risk factors for the development of emerging MDRGN infections (Table 3).

Thus, the prescription of an early and effective antibiotic regimen is crucial for the management of severe MDRGN infections. The available evidence suggests that the greatest benefit of combination antibiotic therapy stems from the increased likelihood of choosing an effective agent during empiric therapy, rather than exploitation of in vitro synergy or the prevention of resistance during definitive treatment. ${ }^{20}$ When a MDR gram-negative pathogen is suspected, the early prescription of a broad-spectrum, combination regimen, followed by a prompt de-escalation upon availability of susceptibility tests should be recommended, looking for a balance between the early start of an effective treatment and the risk of resistance selection.

However, clinicians should be aware that the in vitro susceptibility of the pathogen to the prescribed antimicrobial regimen is not the only point to be considered. The optimization of antimicrobial doses and ways of administration in order to achieve and maintain optimal plasmatic and/or tissue concentrations according with patient's characteristics (age, renal function, obesity) and source of infection are adjunctive crucial elements to be taken into consideration. ${ }^{21,22} \mathrm{~A}$ recent study conducted in several ICU worldwide showed that antimicrobials are routinely underdosed, especially $\beta$-lactams. Probably a paradigm change to more personalized antibiotic dosing may be necessary to improve outcomes for these most seriously ill patients. ${ }^{23}$ The dosages of the different antimicrobial agents are listed in Table 4. An algorithm for the prescription of and adequate empiric treatment for each MDR gram-negative pathogen is proposed in Figure 1.

\section{Targeted treatment}

\section{Extended-spectrum- $\beta$-lactamases-producing Enterobacteriaceae}

The main dilemma in infections caused by ESBLproducing Enterobacteriaceae is the best use of carbapenems. In order to avoid the threat of CRE, carbapenem-saving strategies should be considered for ESBL infections and $\beta$-lactam/ $\beta$-lactamase inhibitor (BLBLIs) combinations, such as piperacillin-tazobactam, have been recommended as an alternative to carbapenems for ESBLs. ${ }^{24}$

Carbapenems are still considered the empirical treatment of choice against serious infections (severely ill patients and/or those with septic shock) caused by ESBL-producing bacteria. ${ }^{2}$ The empirical administration of ertapenem for suspected ESBL Enterobacteriacae is not recommended, since some concerns are rising regarding the isolated in vitro resistance to this drug and the need for further data on severely ill patients. ${ }^{25,26}$

However, according to recent data, BLBLIs, if active in vitro, appear to be as effective as carbapenems for empirical therapy of bloodstream infections due to ESLB-Enterobacteriaceae, regardless of the source and specific species, if used at appropriate doses. ${ }^{27}$ Therefore, high dosage with loading dose and semicontinuous administration of BLBLIs, supported by therapeutic drug monitoring should be preferred for clinically stable patients. ${ }^{2,23}$

Among other $\beta$-lactams, there is still poor information about the efficacy of active cephalosporins and

Table 2. Epidemiology of emerging gram-negative bacteria in USA and Europe.

\begin{tabular}{lccc}
\hline Enzyme & USA (\%) & North-Centre Europe* $\mathbf{( \% )}$ & Italy-Greece (\%) \\
\hline ESBL Escherichia coli & 14 & $5-25$ & $10-50$ \\
\hline ESBL Klebsiella pnemoniae & 23 & $1-25$ & $>50$ \\
\hline CRE-KPC & 11 & $1-5$ & $25-50$ \\
\hline MDR Pseudomonas aeruginosa & 13 & $1-25$ & $25-50$ \\
\hline MDR Acinetobacter & 63 & $1-10$ & $>50$ \\
\hline
\end{tabular}

*North Centre Europe include all European country except Italy and Greece. ESBL, extended-spectrum- $\beta$-lactamase; CRE, carbapenemase-producing Enterobacteriacae; KPC, Klebsiella pneumoniae carbapenemase; MDR, multidrug resistant. 
is likely to result in treatment failure (even with in vitro susceptibility). However, cephalosporin- $\beta$-lactamase inhibitors, namely ceftolozane-tazobactam and ceftazidime-avibactam, are novel agents that appear to have greater activity against ESBL-producing organisms. ${ }^{28}$

\section{Key point}

In conclusion, in our opinion the use of carbapenems may be reserved for patients with severe infections caused by ESBL-producing bacteria, and de-escalation therapy should always be performed if feasible (Table 2). The best alternative to carbapenems for the treatment of ESBL infections is represented by BLBLIs, which can be used in patients with bloodstream infection (BSI) caused by ESBL, especially with lower piperacillin/ tazobactam minimum inhibitory concentration (MIC) (e.g., $\leq 16 / 4 \mu \mathrm{g} / \mathrm{mL}$ ). ${ }^{29,30}$ The use of $\beta$-lactams should be maximized by a pharmacokinetic/pharmacodynamics (PK/PD) point of view with the administration of high dosages and prolonged infusion strategies maximizing the time above the MIC $(\mathrm{t}>\mathrm{MIC})$. A loading dose followed by maintenance doses with extended or continuous infusion is recommended (Table 2).$^{29}$ Although there is less clinical experience to recommend the use of other antibiotics (tigecycline, aminoglycosides, fosfomycin, fluoroquinolones or trimethoprim/sulfamethoxazole), alternative therapeutic approaches could be considered for ESBL-producing Enteobacteriacae based on the susceptibility test results.

\section{Carbapenemase resistant Enterobacteriacae}

Treatment recommendations in CRE infections are mostly based on the accumulating clinical experience from KPC and should be based on several aspects. Combination treatment containing two or three active drugs has shown significant advantages over monotherapy in terms of survival for KPC infections. ${ }^{31-33}$

The role of carbapenems in infections caused by $\mathrm{KPC}$ is still debated. Among the different combinations, high dose carbapenem regimens have been associated with better outcome in previous reports. ${ }^{31,34}$ Tumbarello et al. reported that survival rates for combination regimens that included meropenem were $87 \%$ at meropenem MICs $<4,75 \%$ at MICs of $8 \mathrm{mg} / \mathrm{L}$ and $65 \%$ at MICs $>16 \mathrm{mg} / \mathrm{L}$, which was better than the overall survival rate $(58 \%)$ reported in the study. ${ }^{31}$ Daikos et al. also found that patients with KPC infections who received carbapenem-containing combination regimens had significantly lower mortality rates compared with patients who received non-carbapenem-containing regimens ( 12 vs $41 \%$; $\mathrm{P}=0.006$ ), especially in cases where the MIC of the infecting isolate was $<4 \mathrm{mg} / \mathrm{L} .{ }^{33}$

In keeping with these results, a recent study by Tumbarello et al. supported the use of carbapenems

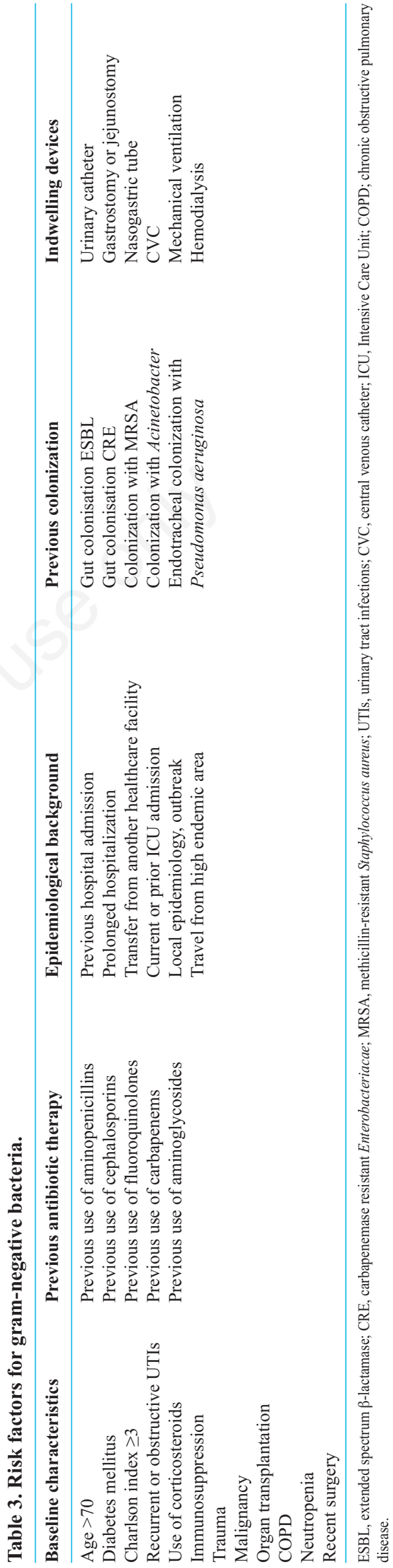


for the treatment of KPC, but with some fundamental conditions, such as low carbapenem MIC for the infecting organism $(\leq 8 \mathrm{mg} / \mathrm{L})$, optimal PK/PD exposure to carbapenem, and combination with another active compound. ${ }^{32}$

Clinical experience with therapeutic drug monitoring (TDM) of $\beta$-lactams for KPC remains scarce, but it is most likely to be beneficial. ${ }^{35}$ For carbapenems, as for other time-dependent agents, the maintenance of concentrations $\left(\mathrm{C}_{\text {trough }}\right)$ above MIC for about $40 \%$ $50 \%$ of the time between dosing interval represents the target for bactericidal activity. In KPC related infections, however, there is the need to maintain a $\mathrm{C}_{\text {trough }}$ level above the MIC for the entire dosing interval. ${ }^{36}$ Pharmacokinetic data have found that high-dosed, prolonged (continuous or extended) infusion of meropenem could achieve adequate exposures $(40 \%$ $\mathrm{C}_{\text {trough }}$ Time $>$ MIC) in 100, 75 and $40 \%$ of septic patients infected with KPC-Kp isolates with MICs of 4, 8 and $16 \mathrm{mg} / \mathrm{L}$, respectively. ${ }^{37-40}$

Antibiotics that permeabilize the bacterial cell membrane (e.g., polymyxins), interfere with cell wall synthesis (e.g., fosfomycin), or inhibit protein synthesis (e.g., aminoglycosides or tigecycline) may decrease the MIC sufficiently so that it is exceeded when a carbapenem is co-administered. Therefore, combination therapy should be strongly considered. ${ }^{41,42} \mathrm{Col}-$ istin is considered a highly active in vitro agent against KPC. ${ }^{43}$ Clinical use of tigecycline for MDR infections has been heterogeneous, but seem to be effective and safe in the treatment of CRE as part of a combination regimen especially when administered at higher doses. ${ }^{44}$ Aminoglycoside-containing regimens (particularly gentamicin) and fosfomycin have also been associated with favourable outcomes and should be encouraged particularly in view of increasing rates of colistin resistance. ${ }^{2,33}$

In addition, the double-carbapenem regimen (ertapenem plus high-dose meropenem or doripenem) has shown to enhance efficacy over either agent alone in previous in vitro and in vivo studies and has been recently considered a possible therapeutic strategy in KPC with high carbapenem MIC or colistin resistance ${ }^{45-47}$ The proposed rationale is that ertapenem has a higher affinity to the KPC enzyme, therefore acting as a suicide substrate and allowing the second carbapenem to be protected from the KCP carbapenemase ${ }^{45-47}$ Controlled clinical data, however, are needed to determine the efficacy of this treatment.

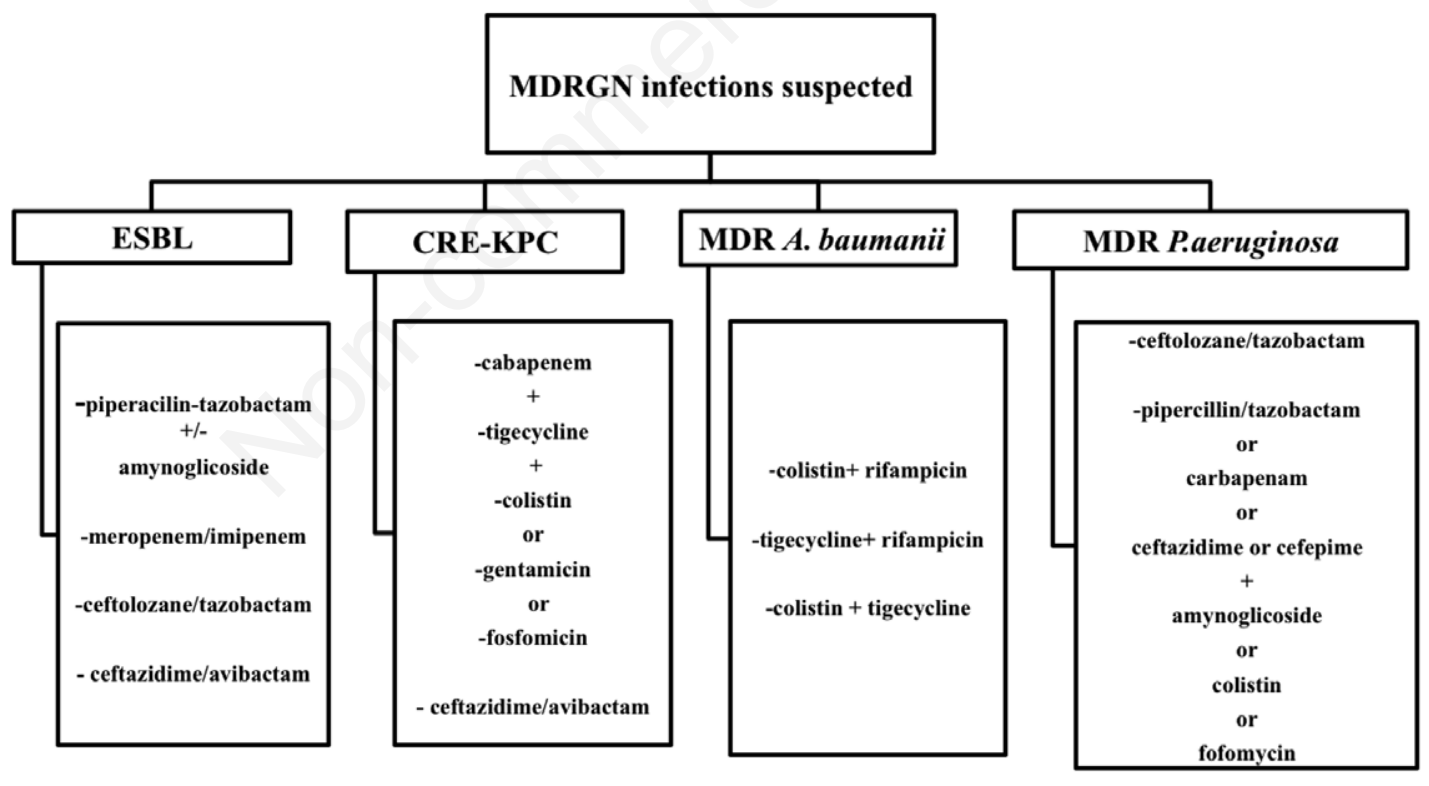

Figure 1. Empirical treatment for suspected multidrug-resistant Gram-negative infections. Choice of antibiotic should be based on suspected site of infection and local penetration. Colistin and aminoglycosides (amikacin and tobramicin) represent good options in respiratory tract infections, however due to the lack of adequate lung penetration inhaled formulations should be preferred (colistin 2 MUI every $8 \mathrm{~h}$ or tobramycin $300 \mathrm{mg}$ every $12 \mathrm{~h}$ or amikacin 500 mg every 12 $h$ ). Intravenous aminoglycosides and colistin should be preferred mainly in bloodstream infections. Intravenous fosfomycin is an option for the treatment of urinary tract infections. Inhaled antibiotic can be associated to empirical intravenous therapy for suspected respiratory tract infections. Metronidazole $500 \mathrm{mg}$ every $8 \mathrm{~h}$ should be associated to ceftolozane/tazobactam or ceftazidim/avibactam for suspected intraabdominal infection. ESBL, extended-spectrum $\beta$ lactamase; CRE-KPC, carbapenemase resistant Enterobacteriacae - Klebsiella pneumoniae carbapenemase; MDR, multidrug resistant; A. baumannii, Acinetobacter baumannii; P. aeruginosa, Pseudomonas aeruginosa. 


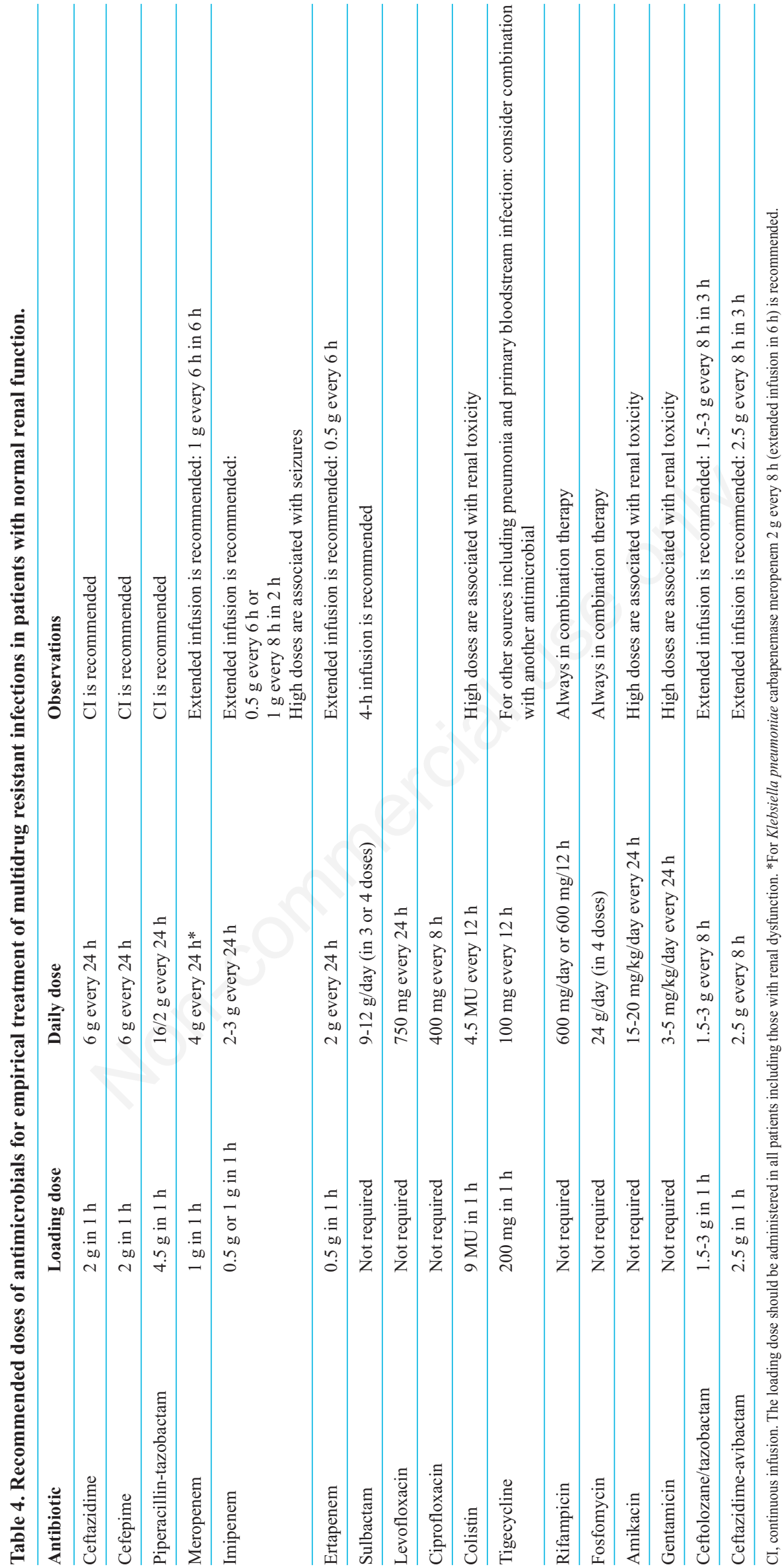




\section{Key point}

In our opinion, in KPC with MIC $\leq 8-16 \mathrm{mg} / \mathrm{L}$ meropenem should be administered at high doses and prolonged infusion in combination regimens (with two antibiotics with in vitro activity), according to TDM. When the MIC is higher than $16 \mathrm{mg} / \mathrm{L}$ carbapenem excluding combination, therapy should be performed considering in vitro activity of antimicrobials. Doublecarbapenem regimen is a possible therapeutic strategy in KPC with colistin resistance or high carbapenem MIC (meropenem MIC >8-16 $\mu \mathrm{g} / \mathrm{Ml}$ ) (Table 5).

\section{Multidrug resistant Acinetobacter}

Adequate therapy of severe infections caused by A. baumannii is crucial in terms of survival. ${ }^{48}$ However, the inherent and acquired resistance of $\mathrm{A}$. baumannii limits the number of antimicrobial options, and makes the selection of an appropriate antimicrobial regimen extremely difficult. Traditionally, carbapenems (except ertapenem) have been considered as the drug of choice for the treatment of A. baumannii infections, in areas with high rates of susceptibility. ${ }^{49}$ In those areas with high rates of carbapenem-resistant $A$. baumannii, carbapenems should not be used, at least in monotherapy. Other therapeutic options include sulbactam, aminoglycosides, polymyxins and tigecycline. ${ }^{50}$ Optimal dosing of sulbactam is unclear and there is concern about the fact that in vitro activity does not necessarily predict clinical outcomes. ${ }^{51}$

Colistin and tigecycline, although active in vitro, are limited in vivo by suboptimal PK characteristics and by the emergence of resistance during therapy. ${ }^{52}$ In strains susceptible to colistin and demonstrating a low MIC for sulbactam $(\leq 4 \mathrm{mg} / \mathrm{L})$, the use of sulbactam may be preferable in the directed therapy based on its better safety profile and to preserve colistin. Tigecycline may be a suitable alternative in the directed therapy for infections of the approved indications caused by MDR A. baumannii if the MIC to this agent is $\leq 1 \mathrm{mg} / \mathrm{L}$ and the isolate is resistant to other agents. However polymyxins have shown the greatest level of in vitro activity against $A$. baumannii and are the most commonly used agents for Acinetobacter isolates resistant to first-line agents; various combination regimens have been considered, mostly with colistin serving as backbone. ${ }^{53}$

In regards to colistin plus rifampin, a large prospective study performed in Italy comparing the use of colistin monotherapy versus colistin plus rifampicin in patients with severe infections due to MDR Acinetobacter. Combination therapy was associated with higher microbiological eradication without an impact on mortality. ${ }^{54}$

The combination of colistin with a carbapenem for the treatment of MDR A. baumannii infections has been analysed only in retrospective studies suggesting that colistin-carbapenem combinations may result in improved clinical responses and survival compared to other regimens and may also limit the emergence of colistin resistance. ${ }^{55}$

The largest comparative study analyzed 250 patients with XDR-Ab BSIs matching colistin monotherapy to combination therapy with colistin + meropenem, col-

Table 5. Drugs recently approved by Food and Drug Administration or in clinical development with activity against multidrug resistant Enterobacteriaceae.

\begin{tabular}{|c|c|c|c|c|}
\hline 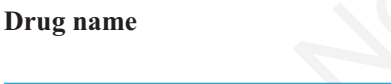 & ESBL & CRE & $\begin{array}{c}\text { MDR } \\
\text { P. aeruginosa }\end{array}$ & $\begin{array}{c}\text { MDR } \\
\text { Acinetobacter }\end{array}$ \\
\hline $\begin{array}{l}\text { Cephalosporin } \\
\text { S-649266 }\end{array}$ & Yes & KPC and NDM-1 & Yes & Yes \\
\hline $\begin{array}{l}\text { Cephalosporin }+\beta \text {-lactamase inhi } \\
\text { Ceftolozane-tazobactam } \\
\text { Ceftazidime-avibactam } \\
\text { Ceftaroline fosamil-avibactam }\end{array}$ & $\begin{array}{l}\text { Yes } \\
\text { Yes } \\
\text { Yes }\end{array}$ & $\begin{array}{l}\text { NO } \\
\text { KPCs and OXA-48 (not active against MBLs) } \\
\text { KPCs and OXA-48 (not active against MBLs) }\end{array}$ & $\begin{array}{l}\text { Yes } \\
\text { Yes } \\
\text { No }\end{array}$ & $\begin{array}{l}\text { No } \\
\text { No }\end{array}$ \\
\hline $\begin{array}{l}\text { Monobactam }+ \text { novel } \beta \text {-lactamase } \\
\text { Aztreonam-avibactam }\end{array}$ & Yes & MBLs such as NDM & Yes & No \\
\hline $\begin{array}{l}\text { Carbapenem }+ \text { novel } \beta \text {-lactamase } \\
\text { Meropenem/vaborbactam } \\
\text { Imipenem/cilastatin-relebactam }\end{array}$ & $\begin{array}{l}\text { Yes } \\
\text { Yes }\end{array}$ & $\begin{array}{l}\text { KPCs } \\
\text { KPCs and OXA-48 (not active against MBLs) }\end{array}$ & $\begin{array}{l}\text { No* } \\
\text { No* }\end{array}$ & $\begin{array}{l}\mathrm{No}^{\circ} \\
\mathrm{No}^{\circ}\end{array}$ \\
\hline $\begin{array}{l}\text { Aminoglycoside } \\
\text { Plazomicin }\end{array}$ & Yes & Most KPCs (not active against many NDMs) & No* & No \\
\hline $\begin{array}{l}\text { Tetracycline } \\
\text { Eravacycline }\end{array}$ & Yes & KPCs & No & Yes \\
\hline
\end{tabular}

ESBL, extended-spectrum $\beta$-lactamases; CRE, carbapenemase resistant Enterobacteriacae; MDR, multidrug resistant; P. aeruginosa, Pseudomonas aeruginosa; KPC, Klebsiella pneumoniae carbapenemase; NDM, New Delhi metallo- $\beta$-lactamase; OXA, oxacillinase; MBL, metallo- $\beta$-lactamase. *Active against $P$. aeruginosa, but not MDR $P$. aeruginosa; ${ }^{\circ}$ active against Acinetobacter baumannii but not against MDR A. baumannii. 
istin + sulbactam and colistin plus other agents. ${ }^{56}$ Outcome in terms of 14-day survival, eradication rate and in-hospital mortality was significantly better among patients receiving combination therapy, although no differences between the 3 different combination regimens were found. Colistin-glycopeptide and colistin-fosfomycin combination has shown to have synergy activity against MDR A. baumannii. ${ }^{57,58}$

\section{Key point}

In conclusion, no definitive recommendation can be made with regard to combination treatment or monotherapy for MDR Acinetobacter infections. Moreover, one potential benefit of combination therapy is the prevention of the emergence of resistance under therapy (especially for colistin and tigecycline).$^{59}$ The combination of sulbactam or a polymyxin with a second agent (tigecycline, rifampicin, or fosfomycin) may be considered for clinical failures or for infections caused by an isolate with MIC in the upper limit of susceptibility.

\section{Multidrug resistant Pseudomonas aeruginosa}

Adequate initial antibiotic treatment is crucial for the management of severe $P$. aeruginosa infections. ${ }^{60,61}$ Recent studies showed that nowadays the prescription of an empiric monotherapy is frequently inadequate in $P$. aeruginosa infections. ${ }^{62}$ Thus, when $P$. aeruginosa is suspected, due to the high rates of antimicrobial resistance, the empiric prescription of a combination therapy should be nowadays encouraged. Several factors, including source of infections and local epidemiology, should be considered in the choice of the optimal empiric combination regimen. The ideal approach consists in the prescription of an antipseudomonal beta-lactam (piperacillin/tazobactam, ceftazidime, or a carbapenem) plus and adjunctive second-agent. ${ }^{63}$

A large meta-analysis compared the effectiveness and safety of $\beta$-lactam combined with aminoglycoside or fluoroquinolone for the treatment of $P$. aeruginosa infection compared with $\beta$-lactam monotherapy. ${ }^{64}$ The evidence suggests that combo regimen does not result in survival benefit; sub-analyses that included patients with bacteraemia, severe or milder infections did not show any benefit with combination therapy. According to these results, inappropriate antibiotic treatment was confirmed to be the main predictor for mortality; therefore, empirical combination therapy should improve outcome since it increases the chance of adequacy.

In an epidemiological setting characterised by the high prevalence of strains of $P$. aeruginosa resistant to fluoroquinolones, the combination of a beta-lactam and an aminoglycoside is probably safer. Once the susceptibility of $P$. aeruginosa has been attested, switching to monotherapy or de-escalating is consid- ered to be safe and effective. De-escalation and prompt discontinuation of antibiotic therapy may lessen the risk of drug-related adverse events, reduce antibiotic pressure on bacterial ecology and finally diminish the emergence of resistant pathogens. ${ }^{65}$

The emergence of multidrug-resistant $P$. aeruginosa strains and the lack of new antimicrobials in the drug development pipeline have renewed the interest in old antibiotics, which had been abandoned for many years because of their excessive toxicity or poor handling. Among these, colistin, which is a cationic and multicomponent lipopeptide belonging to the class of polymyxins has been widely re-entered in clinical use.

According to data published in a prospective cohort study in 2010, due to lower efficacy and tolerability of colistin compared to beta-lactam antibiotics, its use should be restricted to the treatment of infections caused by $P$. aeruginosa resistant to other more active antibiotics. ${ }^{66}$

\section{Key point}

In conclusion, defining the best strategy for empirical treatment of patients at risk of MDR-P. aeruginosa infection will need further studies. On choosing a combination regimen, clinicians have to balance the risk of a greater toxicity with the emergence of antimicrobial resistance. Although fluoroquinolones have optimal tissue penetration, synergy with beta-lactams and low toxicity, resistances have been steadily increasing. Therefore, the combination of a $\beta$-lactam and an aminoglycoside seems to be safer in the empirical treatment. We recommend initiating empirical therapy with two antipseudomonal agents during the first 3-5 days, while waiting for microbiological results of cultures. If $P$. aeruginosa is isolated, the combination therapy can be de-escalated to monotherapy on the basis of the specific susceptibility pattern of the pathogen. Colistin should be restricted to the treatment of pathogens resistant to other more active molecules. Recently approved antimicrobial compounds including new cephalosporins or novel $\beta$-lactamase inhibitors have considerable potential in this setting.

\section{Inhaled antibiotic therapy for multidrug resistant Gram-negative bacteria}

Inhaled antibiotic therapy is conceptually appealing because of the toxicity and the unsatisfactory PK behavior of both colistin and aminoglycosides in the lung. Published studies suggest that aerosolized antibiotic therapy can achieve sputum and airway concentrations 100-fold greater than the MIC of most bacteria, including many MDR strains, although concentrations in lung tissue or epithelial lining fluid are often lower. ${ }^{67}$ 
Table 6. Hospital infection control measures for multidrug-resistant Gram-negative bacteria.

Bundled interventions

Standard hand hygiene

Early identification and contact precautions for colonized and infected patients

Active surveillance and isolation (until culture results are available) for previously colonized or infected with MDRGN patients recently treated at endemic institutions asymptomatic carriers during outbreaks

Cohort nursing staff

Antimicrobial stewardship policies

Education programmes

Cleaning of environmental surfaces

Decolonization of patients (chlorhexidine gluconate baths)

Gut decolonization

MDRGN, multidrug-resistant Gram-negative.

\section{New antibiotics}

Novel antimicrobials that could provide clinical efficacy towards MDR Gram-negative pathogens are urgently needed. Food and Drug Administration recently approved two novel combination antibiotics, ceftolozane-tazobactam and ceftazidime-avibactam, which has recently been approved for the treatment of complicated intra-abdominal infections (in combination with metronidazole) and complicated urinary tract infections. Both these combination agents have been shown in vitro activity against selected resistant Gramnegative pathogens, including Enterobacteriaceae and P. aeruginosa. ${ }^{68}$

The antipseudomonal activity of ceftolozane results from its ability to evade multiple resistance mechanisms, including efflux pumps, reduced uptake through porin channels, and modification of penicillin-binding proteins. ${ }^{69}$ The development of resistance to ceftolozane tazobactam among strains of $P$. aeruginos $a$ has been reported to be slower than resistance to other antipseudomonal agents, and it remained active against mutants resistant to ceftazidime, ciprofloxacin, and meropenem. ${ }^{70}$ Spectrum of activity of ceftolozane/ tazobactam includes difficult-to-treat Gram-negative pathogens, including ESBL strains. ${ }^{28,71-73}$

Another new therapeutic option is represented by ceftazidime/avibactam, where the new $\beta$-lactam inhibitor agent avibactam improves the activity of ceftazidime against MDR P. aeruginosa..$^{74}$ Notably, ceftazidime/avibactam has demonstrated consistent activity against KPC-Kp. ${ }^{28,75}$ (Table 5). Other inhibitor combinations that have at least completed phase 1 clinical trials are ceftaroline fosamil/avibactam, imipenem/relebactam, meropenem/RPX7009.

\section{Conclusions}

For the next years, while waiting for new therapeutic options, it is essential that the last remaining antimicrobials be safeguarded through rational selection and improved infection control. Antibiotic stewardship programs should be developed and implemented by local and international interventions.

It should translate into the implementation of specific guidelines, targeted at education to optimize choice, dosage, and duration of antibiotics in order to improve outcomes and reduce the development of resistance. Moreover, as unmet medical needs call for the use of existing antibiotics in no approved indications or higher-than-approved dosages, regulations will need to be flexible and be updated following the evidence coming from nonrandomized trials.

Infection control protocols are another essential component for reducing the transmission of resistance. The key components of a successful hospital bundle for MDRGN are yet to be determined but have been successful at reducing nosocomial infection rates and controlling outbreaks. Most include improved hand hygiene compliance, contact precaution, control interventions cohort, staff education, and environmental disinfection $^{76-80}$ (Table 6). Other infection prevention strategies such as decolonization of patients by the use of daily chlorhexidine bathing have also demonstrated a reduction in the acquisition of MDR Gram-negative bacteria. ${ }^{78}$ In addition, selective decontamination strategies have shown to be safe and possibly effective during therapy, but success at decolonization may favour the emergence of resistant strains and longterm effects are unclear. ${ }^{81}$ 


\section{References}

1. Zilberberg MD, Shorr AF, Micek ST, et al. Multi-drug resistance, inappropriate initial antibiotic therapy and mortality in Gram-negative severe sepsis and septic shock: a retrospective cohort study. Crit Care 2014;18:596.

2. Bassetti M, De Waele JJ, Eggimann P, et al. Preventive and therapeutic strategies in critically ill patients with highly resistant bacteria. Intens Care Med 2015; 41:776-95

3. Hendrik TC, Voor In't Holt AF, Vos MC. Clinical and molecular epidemiology of extended-spectrum beta-lactamase-producing Klebsiella spp.: a systematic review and meta-analyses. PLoS One 2015;10:e140754.

4. Delgado-Valverde M, Sojo-Dorado J, Pascual A, Rodriguez-Bano J. Clinical management of infections caused by multidrug-resistant Enterobacteriaceae. Ther Adv Infect Dis 2013;1:49-69.

5. Antunes NT, Lamoureaux TL, Toth M, et al. Class D beta-lactamases: are they all carbapenemases? Antimicrob Agents Chemother 2014;58:2119-25.

6. Quinn JP. Carbapenemases: a worldwide threat. Expert Rev Anti-Infect Ther 2013;11:643-4.

7. Philippon A, Slama P, Deny P, Labia R. A structure-based classification of class A beta-lactamases, a broadly diverse family of enzymes. Clin Microbiol Rev 2016;29:29-57.

8. Borges-Walmsley MI, McKeegan KS, Walmsley AR. Structure and function of efflux pumps that confer resistance to drugs. Biochem J 2003;376:313-38.

9. Centers for Diseases Prevention and Control (CDC). Antibiotic resistance threats in the United States, 2013. Available from: http://www.cdc.gov/drugresistance/threatreport-2013/

10. European Centre for Disease Prevention and Control (ECDC). Antimicrobial resistance surveillance in Europe 2014. Annual Report of the European Antimicrobial Resistance Surveillance Network (EARS-Net). Stockholm: ECDC; 2015. Available from: http://ecdc. europa.eu/en/publications/Publications/antimicrobial-resistance-europe-2014.pdf

11. Huang Y, Yu X, Xie M, et al. Widespread dissemination of carbapenem-resistant Escherichia coli sequence type 167 strains harboring blaNDM-5 in clinical settings in China. Antimicrob Agents Chemother 2016;60:4364-8.

12. Dortet L, Cuzon G, Nordmann P. Dissemination of carbapenemase-producing Enterobacteriaceae in France, 2012. J Antimicrob Chemother 2014;69:623-7.

13. Vincent JL, Rello J, Marshall J, et al. International study of the prevalence and outcomes of infection in intensive care units. JAMA 2009;302:2323-9.

14. Tabah A, Koulenti D, Laupland K, et al. Characteristics and determinants of outcome of hospital-acquired bloodstream infections in intensive care units: the EUROBACT International Cohort Study. Intens Care Med 2012;38:1930-45.

15. Martin-Loeches I, Torres A, Rinaudo M, et al. Resistance patterns and outcomes in intensive care unit (ICU)-acquired pneumonia. Validation of European Centre for Disease Prevention and Control (ECDC) and the Centers for Disease Control and Prevention (CDC) classification of multidrug resistant organisms. J Infect 2015;70:213-22.

16. Gudiol C, Royo-Cebrecos C, Laporte J, et al. Clinical features, aetiology and outcome of bacteraemic pneumonia in neutropenic cancer patients. Respirology 2016 [Epub ahead of print].

17. Baker TM, Satlin MJ. The growing threat of multidrugresistant Gram-negative infections in patients with hematologic malignancies. Leuk Lymph 2016;57:2245-58.

18. Lim CJ, Cheng AC, Kennon J, et al. Prevalence of multidrug-resistant organisms and risk factors for carriage in long-term care facilities: a nested case-control study. J Antimicrob Chemother 2014;69:1972-80.

19. Venkatachalam I, Yang HL, Fisher D, et al. Multidrugresistant gram-negative bloodstream infections among residents of long-term care facilities. Infect Control Hosp Epidemiol 2014;35:519-26.

20. Tamma PD, Cosgrove SE, Maragakis LL. Combination therapy for treatment of infections with gram-negative bacteria. Clin Microbiol Rev 2012;25:450-70.

21. Pai MP, Cojutti P, Pea F. Pharmacokinetics and pharmacodynamics of continuous infusion meropenem in overweight, obese, and morbidly obese patients with stable and unstable kidney function: a step toward dose optimization for the treatment of severe gram-negative bacterial infections. Clin Pharmacokinet 2015;54:933-41.

22. Bassetti M, Righi E, Carnelutti A. Bloodstream infections in the Intensive Care Unit. Virulence 2016;7:267-79.

23. Roberts JA, Paul SK, Akova M, et al. DALI: defining antibiotic levels in intensive care unit patients: are current beta-lactam antibiotic doses sufficient for critically ill patients? Clin Infect Dis 2014;58:1072-83.

24. Harris PN, Tambyah PA, Paterson DL. Beta-lactam and beta-lactamase inhibitor combinations in the treatment of extended-spectrum beta-lactamase producing Enterobacteriaceae: time for a reappraisal in the era of few antibiotic options? Lancet Infect Dis 2015;15:475-85.

25. Gutierrez-Gutierrez B, Bonomo RA, Carmeli Y, et al. Ertapenem for the treatment of bloodstream infections due to ESBL-producing Enterobacteriaceae: a multinational pre-registered cohort study. J Antimicrob Chemother 2016;71:1672-80.

26. Rodriguez-Bano J, Cisneros JM, Cobos-Trigueros N, et al. Diagnosis and antimicrobial treatment of invasive infections due to multidrug-resistant Enterobacteriaceae. Guidelines of the Spanish Society of Infectious Diseases and Clinical Microbiology. Enferm Infecc Microbiol Clin 2015;33:337e1-e21.

27. Gutierrez-Gutierrez B, Perez-Galera S, Salamanca E, et al. A multinational, preregistered cohort study of beta-lactam/beta-lactamase inhibitor combinations for treatment of bloodstream infections due to extended-spectrum-betalactamase-producing Enterobacteriaceae. Antimicrob Agents Chemother 2016;60:4159-69.

28. van Duin D, Bonomo RA. Ceftazidime/avibactam and ceftolozane/tazobactam: second-generation beta-lactam/beta-lactamase combinations. Clin Infect Dis 2016 [Epub ahead of print].

29. Nguyen HM, Shier KL, Graber CJ. Determining a clinical framework for use of cefepime and betalactam/beta-lactamase inhibitors in the treatment of infections caused by extended-spectrum-beta-lactamaseproducing Enterobacteriaceae. J Antimicrob Chemother 2014;69:871-80.

30. Delgado-Valverde M, Torres E, Valiente-Mendez A, et al. Impact of the MIC of piperacillin/tazobactam on the 
outcome for patients with bacteraemia due to Enterobacteriaceae: the Bacteraemia-MIC project. J Antimicrob Chemother 2016;71:521-30.

31. Tumbarello M, Viale P, Viscoli C, et al. Predictors of mortality in bloodstream infections caused by Klebsiella pneumoniae carbapenemase-producing K. pneumoniae: importance of combination therapy. Clin Infect Dis 2012;55:943-50.

32. Tumbarello M, Trecarichi EM, De Rosa FG, et al. Infections caused by KPC-producing Klebsiella pneumoniae: differences in therapy and mortality in a multicentre study. J Antimicrob Chemother 2015;70: 2133-43.

33. Daikos GL, Tsaousi S, Tzouvelekis LS, et al. Carbapenemase-producing Klebsiella pneumoniae bloodstream infections: lowering mortality by antibiotic combination schemes and the role of carbapenems. J Antimicrob Chemother 2014;58:2322-8.

34. Tzouvelekis LS, Markogiannakis A, Piperaki E, et al. Treating infections caused by carbapenemase-producing Enterobacteriaceae. Clin Microbiol Infect 2014;20: 862-72.

35. Huttner A, Harbarth S, Hope WW, et al. Therapeutic drug monitoring of the beta-lactam antibiotics: what is the evidence and which patients should we be using it for? J Antimicrob Chemother 2015;70:3178-83.

36. Pea F, Viale P, Cojutti P, Furlanut M. Dosing nomograms for attaining optimum concentrations of meropenem by continuous infusion in critically ill patients with severe gram-negative infections: a pharmacokinetics/pharmacodynamics-based approach. Antimicrob Agents Chemother 2012;56:6343-8

37. Lee CS, Doi Y. Therapy of infections due to carbapenem-resistant gram-negative pathogens. Infect Chemother 2014;46:149-64.

38. Qureshi ZA, Paterson DL, Potoski BA, et al. Treatment outcome of bacteremia due to KPC-producing Klebsiella pneumoniae: superiority of combination antimicrobial regimens. Antimicrob Agents Chemother 2012;56:2108-13.

39. Morrill HJ, Pogue JM, Kaye KS, LaPlante KL. Treatment options for carbapenem-resistant Enterobacteriaceae infections. Open Forum Infect Dis 2015;2:ofv050.

40. Daikos GL, Markogiannakis A. Carbapenemase-producing Klebsiella pneumoniae: (when) might we still consider treating with carbapenems? Clin Microbiol Infect 2011;17:1135-41.

41. Doi Y, Paterson DL. Carbapenemase-producing Enterobacteriaceae. Semin Respir Crit Care Med 2015;36:74-84.

42. Perez F, El Chakhtoura NG, Papp-Wallace K, et al. Treatment options for infections caused by carbapenemresistant Enterobacteriaceae: can we apply "precision medicine" to antimicrobial chemotherapy? Exp Opin Pharmacother 2016 [In press].

43. Rizek C, Ferraz JR, van der Heijden IM, et al. In vitro activity of potential old and new drugs against multidrugresistant gram-negatives. J Infect Chemother 2015;21: 114-7.

44. Poulakou G, Bassetti M, Righi E, Dimopoulos G. Current and future treatment options for infections caused by multidrug-resistant Gram-negative pathogens. Future Microbiol 2014;9:1053-69.

45. Oliva A, Gizzi F, Mascellino MT, et al. Bactericidal and synergistic activity of double-carbapenem regimen for infections caused by carbapenemase-producing Klebsiella pneumoniae. Clin Microbiol Infect 2015 [Epub ahead of print].

46. Poirel L, Kieffer N, Nordmann P. In vitro evaluation of dual carbapenem combinations against carbapenemaseproducing Enterobacteriaceae. J Antimicrob Chemother 2016;71:156-61.

47. Cprek JB, Gallagher JC. Ertapenem-containing doublecarbapenem therapy for treatment of infections caused by carbapenem-resistant Klebsiella pneumoniae. Antimicrob Agents Chemother 2015;60:669-73.

48. Zilberberg MD, Nathanson BH, Sulham K, et al. Multidrug resistance, inappropriate empiric therapy, and hospital mortality in Acinetobacter baumannii pneumonia and sepsis. Crit Care 2016;20:221.

49. Bassetti M, Righi E, Esposito S, et al. Drug treatment for multidrug-resistant Acinetobacter baumannii infections. Future Microbiol 2008;3:649-60.

50. Garnacho-Montero J, Dimopoulos G, Poulakou G, et al. Task force on management and prevention of Acinetobacter baumannii infections in the ICU. Intens Care Med 2015;41:2057-75.

51. Viehman JA, Nguyen MH, Doi Y. Treatment options for carbapenem-resistant and extensively drug-resistant Acinetobacter baumannii infections. Drugs 2014;74: 1315-33.

52. Qureshi ZA, Hittle LE, O'Hara JA, et al. Colistin-resistant Acinetobacter baumannii: beyond carbapenem resistance. Clin Infect Dis 2015;60:1295-303.

53. Garnacho-Montero J, Ortiz-Leyba C, Jimenez-Jimenez FJ, et al. Treatment of multidrug-resistant Acinetobacter baumannii ventilator-associated pneumonia (VAP) with intravenous colistin: a comparison with imipenem-susceptible VAP. Clin Infect Dis 2003;36:1111-8.

54. Durante-Mangoni E, Signoriello G, Andini R, et al. Colistin and rifampicin compared with colistin alone for the treatment of serious infections due to extensively drugresistant Acinetobacter baumannii: a multicenter, randomized clinical trial. Clin Infect Dis 2013;57:349-58.

55. Shields RK, Clancy CJ, Gillis LM, et al. Epidemiology, clinical characteristics and outcomes of extensively drugresistant Acinetobacter baumannii infections among solid organ transplant recipients. PLoS One 2012;7:e52349.

56. Batirel A, Balkan II, Karabay O, et al. Comparison of colistin-carbapenem, colistin-sulbactam, and colistin plus other antibacterial agents for the treatment of extremely drug-resistant Acinetobacter baumannii bloodstream infections. Eur J Clin Microbiol Infect Dis 2014;33:1311-22.

57. Petrosillo N, Giannella M, Antonelli M, et al. Clinical experience of colistin-glycopeptide combination in critically ill patients infected with Gram-negative bacteria. Antimicrob Agents Chemother 2014;58:851-8.

58. Sirijatuphat R, Thamlikitkul V. Preliminary study of colistin versus colistin plus fosfomycin for treatment of carbapenem-resistant Acinetobacter baumannii infections. Antimicrob Agents Chemother 2014;58:5598-601.

59. Poulikakos P, Tansarli GS, Falagas ME. Combination antibiotic treatment versus monotherapy for multidrugresistant, extensively drug-resistant, and pandrug-resistant Acinetobacter infections: a systematic review. Eur J Clin Microbiol Infect Dis 2014;33:1675-85.

60. Micek ST, Lloyd AE, Ritchie DJ, et al. Pseudomonas aeruginosa bloodstream infection: importance of appro- 
priate initial antimicrobial treatment. Antimicrob Agents Chemother 2005;49:1306-11.

61. Pena C, Suarez C, Tubau F, et al. Carbapenem-resistant Pseudomonas aeruginosa: factors influencing multidrugresistant acquisition in non-critically ill patients. Eur J Clin Microbiol Infect Dis 2009;28:519-22.

62. Garnacho-Montero J, Sa-Borges M, Sole-Violan J, et al. Optimal management therapy for Pseudomonas aeruginosa ventilator-associated pneumonia: an observational, multicenter study comparing monotherapy with combination antibiotic therapy. Crit Care Med 2007;35:1888-95.

63. Tumbarello M, Repetto E, Trecarichi EM, et al. Multidrug-resistant Pseudomonas aeruginosa bloodstream infections: risk factors and mortality. Epidemiol Infect 2011;139:1740-9.

64. Vardakas KZ, Tansarli GS, Bliziotis IA, Falagas ME. Beta-lactam plus aminoglycoside or fluoroquinolone combination versus beta-lactam monotherapy for Pseudomonas aeruginosa infections: a meta-analysis. Int J Antimicrob Agents 2013;41:301-10.

65. Bassetti M, Paiva JA, Masterton RG. The case for deescalation in antimicrobial therapy: time to change our strategy in the management of septic shock? Intens Care Med 2014;40:284-5.

66. Paul M, Bishara J, Levcovich A, et al. Effectiveness and safety of colistin: prospective comparative cohort study. J Antimicrob Chemother 2010;65:1019-27.

67. Zampieri FG, Nassar AP Jr, Gusmao-Flores D, et al. Nebulized antibiotics for ventilator-associated pneumonia: a systematic review and meta-analysis. Crit Care 2015; 19:150.

68. Liscio JL, Mahoney MV, Hirsch EB. Ceftolozane/ tazobactam and ceftazidime/avibactam: two novel betalactam/beta-lactamase inhibitor combination agents for the treatment of resistant Gram-negative bacterial infections. Int J Antimicrob Agents 2015;46:266-71.

69. Zhanel GG, Chung P, Adam H, et al. Ceftolozane/ tazobactam: a novel cephalosporin/beta-lactamase inhibitor combination with activity against multidrug-resistant gram-negative bacilli. Drugs 2014;74:31-51.

70. Cabot G, Bruchmann S, Mulet X, et al. Pseudomonas aeruginosa ceftolozane-tazobactam resistance development requires multiple mutations leading to overexpression and structural modification of AmpC. Antimicrob Agents Chemother 2014;58:3091-9.

71. Wagenlehner FM, Umeh O, Steenbergen J, et al. Ceftolozane-tazobactam compared with levofloxacin in the treatment of complicated urinary-tract infections, including pyelonephritis: a randomised, double-blind, phase 3 trial (ASPECT-cUTI). Lancet 2015;385:1949-56.
72. Solomkin J, Hershberger E, Miller B, et al. Ceftolozane/ tazobactam plus metronidazole for complicated intra-abdominal infections in an era of multidrug resistance: results from a randomized, double-blind, phase 3 trial (ASPECT-cIAI). Clin Infect Dis 2015;60:1462-71.

73. Xiao AJ, Miller BW, Huntington JA, Nicolau DP. Ceftolozane/tazobactam pharmacokinetic/pharmacodynamic-derived dose justification for phase 3 studies in patients with nosocomial pneumonia. J Clin Pharmacol 2016;56:56-66.

74. Torrens G, Cabot G, Ocampo-Sosa AA, et al. Activity of ceftazidime-avibactam against clinical and isogenic laboratory pseudomonas aeruginosa isolates expressing combinations of most relevant beta-lactam resistance mechanisms. Antimicrob Agents Chemother 2016;60: 6407-10.

75. Lucasti C, Popescu I, Ramesh MK, et al. Comparative study of the efficacy and safety of ceftazidime/avibactam plus metronidazole versus meropenem in the treatment of complicated intra-abdominal infections in hospitalized adults: results of a randomized, double-blind, Phase II trial. J Antimicrob Chemother 2013;68:1183-92.

76. Pelat C, Kardas-Sloma L, Birgand G, et al. Hand hygiene, cohorting, or antibiotic restriction to control outbreaks of multidrug-resistant Enterobacteriaceae. Infect Control Hosp Epidemiol 2016;37:272-80.

77. De Rosa FG, Corcione S, Cavallo R, et al. Critical issues for Klebsiella pneumoniae KPC-carbapenemase producing K. pneumoniae infections: a critical agenda. Future Microbiol 2015;10:283-94.

78. Hayden MK, Lin MY, Lolans K, et al. Prevention of colonization and infection by Klebsiella pneumoniae carbapenemase-producing enterobacteriaceae in long-term acute-care hospitals. Clin Infect Dis 2015;60:1153-61.

79. Viale P, Tumietto F, Giannella M, et al. Impact of a hospital-wide multifaceted programme for reducing carbapenem-resistant Enterobacteriaceae infections in a large teaching hospital in northern Italy. Clin Microbiol Infect 2015;21:242-7.

80. Otter JA, Yezli S, Schouten MA, et al. Hydrogen peroxide vapor decontamination of an intensive care unit to remove environmental reservoirs of multidrug-resistant gram-negative rods during an outbreak. Am J Infect Control 2010;38:754-6.

81. Bar-Yoseph H, Hussein K, Braun E, Paul M. Natural history and decolonization strategies for ESBL/carbapenem-resistant Enterobacteriaceae carriage: systematic review and meta-analysis. J Antimicrob Chemother 2016 [Epub ahead of print]. 